and introduced quarks, charm etc. $\mathrm{He}$ discussed many models and finished by illustrating how the basic ideas are quite unifying for physics as such, since most of them have been used before.

D.H. Wilkinson, gave a lecture entitled "Elementary particles and nuclei" in which he presented a strong case for nuclear physics as an important tool in the investigation of elementary particles. He did so by talking about two sets of $\beta$ - decay experiments only and succeeded thereby in giving an excellent background setting for the following speaker (Bell). His first argument was based on conservation of the vector current, on the $\mu-e$ universality in the Cabibbo sense and on the Salam-Weinberg unification of weak and electromagnetic interactions. The experimental data are the $\mathrm{ft}$ values for $\mathrm{O}^{+} \rightarrow 0^{+}$superallowed $\beta$ - transitions, the muon $\mathrm{ft}$ value and the Cabibbo angle. An estimate of the mass of the neutral boson obtained from neutral current investigations is also necessary. Putting all this together one obtains a value for the average electric charge of the quarks. The result is: $\mathrm{Q}=(.20 \pm .10) \mathrm{e}$ in agreement with $1 / 2(2 / 3-1 / 3)=.167$. His second result was a limit on the strength of possible second class currents in the Fermi interaction by $\mathrm{ft}$ value comparison for $\beta^{+}$and $\beta^{-}$mirror transitions inside the same isospin multiplet. The result is again as precise as the best high energy result ( $\Sigma$ - meson decay).

In addition nuclear physics had its own sessions organized by $\mathrm{M}$. Petrascu (Bucharest). A.M. Baldin gave a plenary session talk on heavy ion physics with a good resume of one very important line of research in Dubna and with a repeated invitation for others to come and participate. The main speakers in the afternoon sessions were Z. Szymanski who talked about the possible existence of new elements or of exotic states of atomic nuclei, and S.G. Nilsson who gave a lecture on the recently discovered high angular momentum states and the types of effective optical potentials which are needed for the description of such states. There were also lectures by W. Greiner and $H$. Bethge on heavy ion physics, the first from the point of view of nuclear physics including shock waves in nuclear matter and the second from the point of view also of technological applications.

O.K.-H

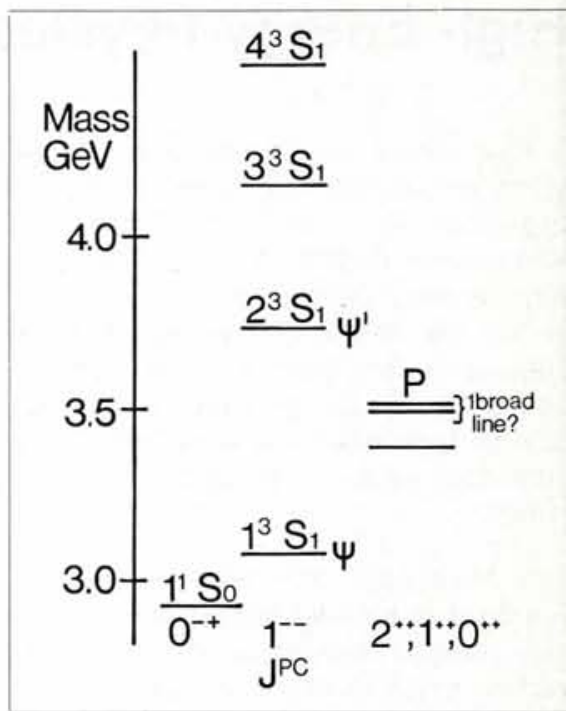

Fig. 3. - The level scheme of the new particles as predicted by the quark model including the special quantum number called charm.

\title{
Energy Strategies
}

The three plenary lectures, directly related to Energy Strategies were given by Haefele (Laxenburg) on "Energy Strategies", Weinberg (Oak Ridge) on "The Maturity of Nuclear Energy", Braams (Jutphaas) on "State of Thermonuclear Research" while the speech by Ursu on "Energy Today: New Goals and Challenges for Physics" was mainly directed at the present knowledge and more local situations. The lectures of the plenary sessions will be published in the proceedings of the conference, therefore it seems appropriate to give in this short report a somewhat detailed review of the subjects discussed in the two afternoon panel sessions.

It is obvious that sufficient food and energy are the two primary requirements mankind is facing. In those regions of the world where large populations are living due to acceptable climatic conditions sufficient arable land is also available, otherwise no population growth would have taken place. Today it is possible to live in many parts of the world with an even larger population and still produce sufficient food if sufficient fertiliser (nitrogen) is available. This means that energy is needed for the production of fertiliser. Furthermore the land has to be ploughed. This requires energy for the production of the construction materials of the tractors anid energy for their operation. The rest is done by photosynthesis. For the provision of reasonable housing and transport the same is true. In the lecture by Haefele many such relations were shown.

In different parts of the world the local energy supply possibilities as well as the energy requirements determine the energy policies. The attitude of the USA with its still reasonably large energy resources with respect to coal, oil and gas as well as nuclear energy tends to be as far as possible independent of outside supplies.

In Western Europe such a possibility does not exist hence it seems very difficult to come to one common energy strategy.
In the first afternoon meeting of the two panel sessions, Weinberg (Oak Ridge) and Hemans (Arnhem) introduced short surveys on Energy Research for the year 2000 and in relation to this long term goal on the development of thermal breeders. It was interesting that both lecturers had the opinion that thermal breeding either with a molten salt system or with an aqueous homogeneous suspension system, should receive more support and stimulation, realizing the importance of thermal breeding next to fast breeding. It should be of interest for a group of experts in this field to review the possibilities and perhaps recommend what to do or not to do.

Two international organizations Unesco (Berkovsky) and the OECD (Drilhon) also presented introductions about their activities in the field. It is of real importance that such international organizations are presenting a platform for mutual discussion among all interested countries in the world. Also the second world wide 
organization, the Vienna Agency should be mentioned in this respect. The director general Eklund informed the conference on the Vienna Agency activities, they being interested in all aspects of nuclear energy and its safety.

On the second afternoon very active discussions among the panel members as well as between panel and audience took place. The following views were presented by the panel :

1) Gheorghe from the Bucharest Polytechnical Institute presented a system for "Structural modelling for Energy Strategies".

Thereafter more pragmatic subjects were introduced by:

2) Mayer-Abich (Essen) on "Ecological aspects of energy research".

3) Decken (Jülich Nuclear Centre) on "Assessment of energy sources and sinks".

4) Colomb (President of the European Nuclear Society, Lausanne) on "Economic aspects of energy production".

5) Kurti (Oxford) on "Technologies of efficient energy utilization".

\section{Conclusions}

The Conference was a time for airing the problems rather than formulating new strategies but the many thoughtful contributions gave proof that a great deal of attention is now being paid to thinking out ways of meeting the world's growing energy demand. Planning is accepted as a real necessity even in the market economy countries as it is recognized that the concept of a free market in this field is already largely illusory.

In practice, the supply of energy to the ultimate user is effectively a monopolistic business either because only a single supplier exists or because the present primary sources are rigidly controlled. Consequently it is desirable in any strategy for the future that real choices are bulit in, to provide the necessary competition and to guard against an unexpected reduction in vital supplies. Unit size will continue to increase with demand, as in the past where the unit size of, for example, central power stations and oil tankers has matched the increase in the use of electricity and the growing oil consumption.

At the same time, however, there should be real attention paid to diversification and the use of localized converters where this is practicable. The essence of the problem here is one of social stability and political maturity.
To plan, it is necessary to have scenarios but before defining scenarios it is equally necessary to define the objective behind the scenarios. The aims expressed by ERDA of seeking independence, international cooperation, maintaining full employment with a healthy economy and respecting the environment will meet with general approbation if independence is interpreted in a non-nationalistic sense. These are however difficult to reconcile all at the same time and need to be quantified if they are to be balanced with any confidence. It is evident that much of the data is either not available or inevitably "fuzzy" but this does not mean that computation is excluded. On the contrary model building and mathematical analysis are essential to understand the interplay of the many factors that need to be taken into account and equally to reveal what choices can be made.

The real limitations must be recognized and not left to emotional evaluation. The environment, perhaps belatedly, is a vital factor in the equation but its price should not be allowed to exceed its economic worth. The amount of attention that can be paid to the many facets of preparing strategies is limited in human terms and there is a danger in paying undue attention to one aspect, of distracting attention from another of possibly greater importance. It is relatively recently that it has been concluded that although the pollution caused by burning fossil fuels could be remedied at a price, even with a huge investment in nuclear power the $\mathrm{CO}_{2}$ buildup in the upper atmosphere will reach a dangerous level. But before that, it is considered by some that the thermal pollution would have begun to cause unacceptable changes in climatic conditions.

Evidently the data upon which such alarming conclusions are based has to be examined with the utmost care. Only the most rigorous scientific evaluation is of any use, carried out with sufficient means and with the widest international collaboration. Good research was a sine qua non of establishing strategies and in its turn formed an integral part of the strategy. It was necessary to know how best to deploy the research effort available and it was not enough simply to allocate funds on the assumption that the one was proportional to the other.

Synthesising the results was also far from easy as the scientific conclusions were likely to be unpalatable to those who had to act upon them. A fundamental look at our wasteful way of transforming energy, which resulted in the liberation of so much heat relative to the work done was going to have far-reaching consequences. Encouragements to use less would only touch the fringe; complete new systems were needed involving transformation, transport, storage anid utilization. There were obvious areas where a major effort could make a major difference as for example in the heating of homes with the installation of hot water grids or individual solar plants but only at a cost.

Regardless of the economy style, the cost (and the implicit energy required to bring about the change) had to be reckoned with. In the words of one participant "cash is shorter than energy" and the basic economics of change could not just be left to market forces but this did not alter the fact that the substitution of one supply for another required an economic incentive. It must be expected that the penetration of a new source into the energy scene will follow classical rules even though the quality of life and limitations to growth are additional parameters in the equation.

The strategies adopted will no doubt vary from region to region and there will be a fair diversity of success as measured by the yard-sticks that future generations will adopt. But strategies can not be left to chance. It is up to science to provide the input data, undertake the necessary research and provide the tools that are required, whilst it is up to the people to establish the priorities and goals and finally exert the will to see them achieved. J.J.W. E.N.S.

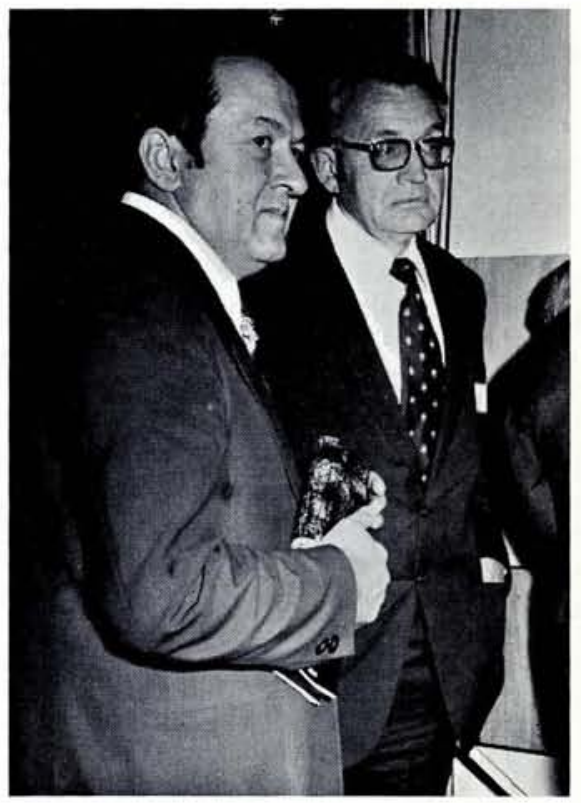

I. Ursu (left) President of the Conference Board with H.B.G. Casimir, President EPS. 